\title{
Pressure Induced Changes in Grain Boundary Conditions of Lithium Conducting Ceramics Characterized by Impedance Spectroscopy
}

John W. Ostrander ( $\square$ john.ostrander@gmail.com )

University of Tulsa Dept. of Chemistry and Biochemistry https://orcid.org/0000-0002-0268-224X

\section{Carolyn Torres}

University of Tulsa Dept. of Chemistry and Biochemistry

\section{Fride Vullum-Breuer}

Norwegian University of Science and Technology

\section{Dale Teeters}

University of Tulsa Dept. of Chemistry and Biochemistry

\section{Research Article}

Keywords: Pressure, Grain Boundary Conditions, Lithium Conducting Ceramics, Impedance Spectroscopy, Solid state batteries, lithium ion

Posted Date: June 25th, 2021

DOl: https://doi.org/10.21203/rs.3.rs-646054/v1

License: (c) (i) This work is licensed under a Creative Commons Attribution 4.0 International License. Read Full License 


\title{
Pressure Induced Changes in Grain Boundary Conditions of Lithium Conducting Ceramics Characterized by Impedance Spectroscopy
}

\author{
John W. Ostrander*1, Carolyn Torres ${ }^{1}$, Fride Vullum-Breuer ${ }^{2}$, and Dale Teeters ${ }^{1}$ \\ 1. University of Tulsa Dept. of Chemistry and Biochemistry, 800 South Tucker Tulsa OK 74104 \\ 2. Norwegian University of Science and Technology, Dept. of Materials Science and Engineering, Trondheim \\ Norway \\ *Corresponding author email john.ostrander@gmail.com; Dale Teeters; dale-teeters@utulsa.edu; Fride \\ Vullum-Breuer, fride.vullum.bruer@sintef.no; Carolyn Torres, cdb524@utulsa.edu
}

\begin{abstract}
$\underline{\text { Abstract }}$
Solid state batteries, particularly for lithium ion based architecture have been the focus of development for over 20 years and are receiving even more attention today. Utilizing impedance spectroscopy (IS) measurements we investigate the response of conductivity versus incremental pressure increase by a piston-cylinder-type high pressure cell up to $1 \mathrm{GPa}$ for some lithium conducting ceramics: LATP $\left(\mathrm{Li}_{1.3} \mathrm{Al}_{0.3} \mathrm{Ti}_{1.7}\left(\mathrm{PO}_{4}\right)_{3}\right)$, LLTO $\left(\mathrm{Li}_{5} \mathrm{La}_{3} \mathrm{Ta}_{2} \mathrm{O}_{12}\right)$, LLT $\left(\mathrm{Li}_{0.33} \mathrm{La}_{0.55} \mathrm{TiO}_{3}\right)$, LAGP $\left(\mathrm{Li}_{1.5} \mathrm{Al}_{0.5} \mathrm{Ge}_{1.5} \mathrm{P}_{3} \mathrm{O}_{12}\right)$ and $\mathrm{LLZO}\left(\mathrm{Li}_{7} \mathrm{La}_{3} \mathrm{Zr}_{2} \mathrm{O}_{12}\right)$ for non-annealed and annealed samples.

Isothermal, incremental pressure increase of powders allows for an in situ observation of the transition state conditions of poorly consolidated ceramic powders and the effects on grain boundary conditions prior to sintering. Specific conductance $\left(\sigma_{b}\right)$ increased by several orders of magnitude in some samples, approaching $10^{-3} \mathrm{~S} \cdot \mathrm{cm}^{-1}$, yet decreased in other samples. The affect of grain boundaries and affects of bulk capacitance as the sample dimensions are altered due to pressure, are attributed to some of this behavior and will be discussed. The understanding of some of these fundamental processes may be valuable in facilitating these and similar ceramics for use in commercial solid state battery systems.
\end{abstract}

\section{Introduction}

Lithium ion batteries have been in widespread production and use in personal electronics since the early 1990s. Since then advances have occurred incrementally, with improved performance, higher energy density, and smaller battery size. Batteries that once were quite large are now very condensed, while maintaining the same power and energy storage as much larger cells. This is desirable for lightweight portable electronics, but with the side effect of improved performance these much smaller batteries are doing the same amount of work as their larger forerunners and heat evolution is an unavoidable consequence. Battery ignition, fires, and explosions of 
lithium cells has resulted in many changes in policy, regulations, and law, notably that free lithium batteries are not permitted in carry on bags with most airlines.

Other considerations are the affects of pressure and the affects on battery degradation under load which would be important with the development of commercial solid state battery systems.

Several solutions have resulted from this problem such as increased interest in the areas of inflammable electrolytes, better battery design, and solid state lithium batteries among others. It is widely stated in publications over the last several years that solid state batteries (SSB) are one solution for problems lithium ion batteries (LIB) such as dendrite formation, over heating, etc. Generally speaking, solid state batteries are as not widely available, and only recently emerging on the commercial market most notably with electric vehicles.

Here, we look at five commercially available lithium containing ceramics as candidates for the solid electrolyte for $\mathrm{SSB}^{1-2}$. As a solid state electrolyte, ceramics are better suited for higher temperatures ${ }^{3,4}$ which can simultaneously address the issue of safety as well as performance.

Investigated in this work are garnets LLTO $\left(\mathrm{Li}_{5} \mathrm{La}_{3} \mathrm{Ta}_{2} \mathrm{O}_{12}\right)^{5-8}$ and $\mathrm{LLZO}\left(\mathrm{Li}_{7} \mathrm{La}_{3} \mathrm{Zr}_{2} \mathrm{O}_{12}\right)^{9}$, LATP (NASICON ${ }^{10}$ structure), LAGP( $\left(\mathrm{Li}_{1.5} \mathrm{Al}_{0.5} \mathrm{Ge}_{1.5} \mathrm{P}_{3} \mathrm{O}_{12}\right)$, and perovskite LLT $\left(\mathrm{Li}_{0.33} \mathrm{La}_{0.55} \mathrm{TiO}_{3}\right)^{11}$. In an effort to reconcile grain boundary conditions and resulting affects on ion conductivity $\left(\sigma_{b}\right)$, each powder was analyzed by impedance spectroscopy in situ to observe changes in grain boundary conditions. The grain boundary phase ${ }^{12}$ may be detected separately ${ }^{8,12}$, or inclusively ${ }^{13}$, but it was not found that in situ studies of grain boundaries have been characterized in this way. We should first define the grain boundary as not only the area of grain-grain contact, but also the adjacent regions and voids where grains are in very close proximity without touching. This definition of grain boundaries has been inferred previously in other publications ${ }^{14-15}$. For simplicity this interpretation is used here as these grain boundaries are variable and expected to change (along with sample thickness) under increased pressures.

Identified in $1968^{16}$, LATP belongs to space group $\mathrm{R} 3 \mathrm{C}^{17-18}$. Considered a super-ionic conductor $^{19}$ and air $\operatorname{stable}^{20}$, the crystal structure most similar to NASICON ( $\mathrm{Na}_{1+\mathrm{x}} \mathrm{Zr}_{2} \mathrm{Si}_{\mathrm{x}} \mathrm{P}_{3-\mathrm{x}} \mathrm{O}_{12}, 0$ $<\mathrm{x}<3)^{21}$ which has been studied intensively since the 1980s for use in sodium or lithium sulfur batteries $^{18,22}$. LATP has also been described as rhombehedral ${ }^{23}$. Ion migration pathways in LATP have been modeled for both sodium ${ }^{24}$ and lithium ${ }^{25,14}$ so much is already known about the bulk ceramic and single crystal form. LAGP also has a NASICON-like structure ${ }^{26}$, sometimes referred to as LISICON ${ }^{25}$ and is less studied than LATP. LATP and LAGP are thus promising due to the super ionic conduction and resistance to lithium metal ${ }^{2,20}$. Conversely LATP Lithium reactions have also 
been reported ${ }^{5,20,27}$ leaving the door open to additional characterization. The presence of trivalent metal ions in the structure can improve bulk ion conductance $\left(\sigma_{b}\right)^{28-31}$ but this is not expected to be the case here.

LLZO as received has a garnet structure. When calcined at $1150^{\circ} \mathrm{C}$ or by other methods $\mathrm{s}^{32-33}$ LLZO recrystalizes to a cubic phase (c-LLZO) ${ }^{20,34-35}$. The cubic phase is considered a super ion conductor $^{7}$, but is less stable than the garnet phase and was not investigated here. Doping of LLZO with aluminum is possible and has been shown to improve conduction ${ }^{29}, 36$, but was avoided here with the use of quartz sample boats for heating.

First mentioned in literature in $1993^{37}$, LLTO is considered an exceptional ion conductor ${ }^{5}$ that is temperature independent ${ }^{38}$. Because of high grain boundary impedance $\left(Z_{\mathrm{GB}}\right)$, some say that LLTO is a better insulator than it is an ion conductor ${ }^{39}$. Contrarily, Sakamaki et al report that partial grain boundaries have higher current flow, and the high grain boundary resistance can be overcome $^{40}$.

Perovskite LLT has been well characterized as a bulk material ${ }^{11}$ and having high $\sigma_{\mathrm{b}}$, but also dominated by grain boundary resistance even under pressure ${ }^{41-45}$. This reasoning is supported by Inaguma et al whom describe elimination of the grain boundary resulting in improved conductance for $\mathrm{LLT}^{46}$. On the other hand, LLT is also reported as reactive with lithium metal ${ }^{46}$.

If lithium metal could be used for anode material, the energy densities of $3860 \mathrm{mAhg}^{-1}$ and oxidation potential of $-3.04 \mathrm{~V}$ vs. SHE would be a significant step in LIB development, but this has so far been a poor candidate due to high reactivity, and dendrite formation ${ }^{47}$. This includes solid state electrolytes ${ }^{48}$ and the solid electrolyte interphase (SEI) formed when using lithium metal anodes.

We hypothesize that by characterizing lithium ion conducting ceramics in situ that we can learn more about the grain boundary interactions and perhaps lay the groundwork for future modeling of these and similar systems. By using commercially available materials, we expect there to be more reproducibility and consistency than experimental or synthesized powders which would add more variables to the study. While used as received, powders were also annealed to relieve internal stressors, and cracks, for a more uniform and optimized powder free from such physical defects as much as possible for additional tests for comparison, while heating below temperatures that would effect recrystallization or sintering.

\section{Materials and methods}




\subsection{Materials}

Ceramics were used as received from Toshima Ltd. (Japan). For sample testing, a KBr pellet press (international crystal laboratories) with vacuum capability was used without modification. Non-porous alumina tubing and E52100 alloy steel were purchased from McMaster Carr. Impedance spectroscopy was taken with a Solartron 1296 interface and a Solartron 1260 gain phase analyzer. Impedance data was analyzed using Zview ${ }^{\circledR}$, and any images were processed in ImageJ 1.50i. Helios Nanolab 600, Fe-SEM (Zeiss Ultra 55 LE, and LV-Fe-SEM (Zeiss Supra 55 VP) were used for SEM imaging, and XRD were conducted on Rigaku XRD. Thermogravimetric (TGA) was performed on TA instrument "Discovery" series TGA in platinum pans. Redundant calculations were performed in Microsoft Excel 2007. Battery cycling was performed with a Maccor 4300M in tandem with a Parstat (Princeton technologies) 4000+. Any glovebox work was conducted inside an MBraun glovebox with typical $\mathrm{O}_{2}$ levels below $5 \mathrm{ppm}$.

\subsection{Methods}

Annealing of powders was done in a tube furnace in alumina boats (except LLZO to avoid Al doping), under argon at specific temperatures for each ceramic. Annealing time was not less than 4 hours for any sample. Reduction of LATP occurred, resulting in the characteristic blue color which was reversible upon subsequent heating in air. The function of annealing reduces average particle size, as explained by Jackman et $a l^{49}$ :

$$
G S_{\text {critical }}=\frac{14.4 \gamma}{E\left(\Delta \alpha_{\max }\right)^{2}(\Delta \mathrm{T})^{2}}
$$

$\gamma_{\mathrm{f}}$ is fracture surface energy, and $\Delta \alpha_{\max }$ is the difference in maximum and minimum principal axis of a unit cell, or grain. Annealing was used in an attempt to procure more pristine grains, and perhaps narrower grain size distribution.

Each sample was subjected to pressure in custom test cell for in situ impedance testing. It was convenient to use a pellet press, originally made for FTIR salt pellets, with a removable alumina tube cut to size was used as an insulator inside the sample holder. Positioning the sample holder and steel shaft reduced incident of alumina tube breakage, but likewise under such pressures cracking of the alumina tube did not compromise the sample, as powders are not fluid and pelletize when subjected to pressure. The polished anvil, part of the pellet press, served as one electrode surface, which was in contact with the metal pellet press, and The hardened steel shaft served as the other 
electrode. The sample holder was set on a blank electronic"bread board" which does not compress, to insulate from the hydraulic press surface.

About 0.2 grams of powder is introduced into the tube. The steel shaft is inserted into the tube, and O-rings were used on both shaft and tube to facilitate vacuum. The hydraulic press was used to apply pressure such that the pressure gauge registered, and was referred to here as $0.00 \mathrm{GPa}$ pressure.

The sample powder was tested under a range of pressure from 0.00 to $1.00 \mathrm{GPa}$, at incremental steps. The pressure forces closer fitting of grains, streamlining the ion migration ${ }^{50}$ by closing the grain-grain voids, and forcing more grain-grain surface contact. Alternatively, this conductance may improve simply by increasing the sample density ${ }^{21}$

\subsection{Impedance Spectroscopy (IS)}

Bulk resistance is derived using equation 2, and the ion conductance by equation 3 . It is important to indicate that ion conductivity is an intrinsic quantity characterizing the entire (bulk) sample. For this particular work the sample thickness, and volume, change with pressure. Bulk resistance $\left(R_{b}\right)$ is described by equation (2) and bulk conductance ( $\sigma$ or $\sigma_{b}$ ) by equation (3):

$$
\begin{aligned}
& \omega R_{b} C_{b}=1 \text { and } R_{b}=\frac{1}{\omega C_{b}} \\
& \sigma=\frac{1}{\mathrm{R}_{\mathrm{b}} \mathrm{A}}, \text { and } \partial \sigma=\frac{\partial \mathrm{l}}{\partial \mathrm{R}_{\mathrm{b}} \mathrm{A}}
\end{aligned}
$$

In equation 2, $\omega=$ angular frequency and $C_{b}=$ bulk capacitance. Equation 3 has two parts, the convention for conductance at constant conditions, and the change in conductance roughly calculated dependent on a change of sample thickness $(\partial \mathrm{l})$, and resistance, $\partial \mathrm{R}_{\mathrm{b}}$. A is electrode surface area in contact with sample, and 1 is sample thickness.

Determination of sample thickness in situ was determined by the difference of the height of the hydraulic press from the top of the sample holder after settling at each pressure increment, and the height of an empty cell with some pressure applied. A feeler gauges and micrometer were used exhaustively in determination of sample thickness.

Impedance spectra were typically taken from $10 \mathrm{MHz}$ to $0.075 \mathrm{~Hz}$ for each sample, at pressure increments from 0 to $1.00 \mathrm{GPA}$ pressure $(0.75 \mathrm{GPa}$ was used in some samples, higher pressures were not always sustainable). 
Electric modulus is useful for materials characterization ${ }^{15,51-55}$. Likewise tan $\delta$, and permittivity $\varepsilon^{*}\left(\varepsilon^{*}=\varepsilon^{\prime}-j \varepsilon^{\prime \prime}, j=\sqrt{-1}\right)$ may also be used in this case to characterize the changes in grain boundary conditions and were useful in determining the correct equivalent circuit. Bulk changes in the ceramic powder grains were not observed nor expected below $1 \mathrm{GPa}$.

In part due to scaling, Nyquist plots can be inconvenient to discern phases (figure 2) as the semi circles, or time constants may differ in size by orders of magnitude. Imaginary modulus (M") was used as it can discern the grain boundary phase ${ }^{15}$ as a local maxima (Figure 3), defined by equation 4 .

$$
\begin{aligned}
& M_{\text {max }}^{\prime \prime}=\frac{\mathrm{e}_{0}}{2 \mathrm{C}_{0}} \\
& \text { Also } \mathrm{M}^{\prime \prime}(\omega)=\frac{\varepsilon^{\prime \prime}}{\varepsilon^{\prime 2}+\varepsilon^{\prime \prime 2}}
\end{aligned}
$$

In eq. 4 and $5, \mathrm{e}_{0}=8,854 \mathrm{pF} / \mathrm{m}$ - permittivity in vacuum , $\mathrm{C}_{0}=$ capacitance, and $\varepsilon^{\prime} / \varepsilon^{\prime \prime}$ are real and imaginary permittivity ( $\varepsilon^{\prime}$ is also known as dielectric constant, and $\varepsilon^{\prime \prime}$ as dielectric loss). Due to the three dimensional nature of the materials, it was necessary to construct an equivalent circuit using parameters beyond this work, such as loss tangent, admittance, permittivity and so on with an exact match for this system found using the model in figure 4, with grain and grain boundaries labeled, the electrical contacts (E1 is the polished anvil/ceramic interface, and E2 is the non-polished steel shaft/ceramic interface) were also detectable using IS.

It is described by Behera $e t a l^{55}$ that a shift of $\mathrm{M}^{\prime \prime}$ max to higher or lower frequency domain is a change in dielectric relaxation in agreement with Liu $e a^{56}$ (illustrated by example in figure 3 , LAGP (as received) pressed from 0.00-0.75GPa) who also describe merging of peaks at higher temperatures due to the conduction process. Moreover, as we are attempting to characterize a material that relies on ion hopping for conductance, the usual methods are sometimes inadequate. Interestingly, the work of Ngai and León ${ }^{57}$ strongly supports using electric modulus to characterize the conductance of ions through a disordered solid, and seem to strongly support our findings in this study. Bulk ion hopping defined by Barsoukov and MacDonald as the inverse of the frequency at $\mathrm{M}_{\text {max }}$ is the conductivity relaxation time, $\tau_{\sigma}$. The relationship ${ }^{57}$ can be described by equation 6 , permittivity (dielectric) is defined in equation 7.

$$
\frac{\tau_{i o n}}{\tau_{\sigma}}=\frac{c q^{2} r^{2}}{6 k_{B} T \varepsilon_{0} \varepsilon_{\infty}}
$$




$$
\varepsilon^{*}=M^{*-1}=\varepsilon^{\prime}-j \varepsilon^{\prime \prime}
$$

( $\varepsilon_{\infty}=$ constant, and is the dielectric permittivity of the material, q is charge (1), $k_{B}$ is the Boltzmann constant $\left(8.854 * 10^{-12} \mathrm{~F} / \mathrm{m}\right), \mathrm{c}$ is concentration, $\mathrm{T}$ is temperature, and $\varepsilon_{0}$ all of which are constant) so $\tau_{\text {ion }} \propto r^{2}$, and $\tau_{\sigma}$ is determined by impedence spectra) Thus a shift in $\mathrm{M}^{\prime \prime}{ }_{\text {max }}$ indicated a shift in $\tau_{\text {ion }}$ when $\mathrm{r}$, the mean hopping distance of the ions, is the variable. Similar behavior is seen here, but at isothermal conditions. These frequency dependent changes indicate changes in relaxation times, and activation energies. Arrhenius plots may be calculated by equations 8 and 9

$$
\begin{aligned}
\tau_{\text {peak }} & =\tau_{0} \operatorname{Exp}\left(E a / K_{B} T\right) \\
\tau_{\text {peak }} & =1 / 2 \pi f^{\text {peak }}
\end{aligned}
$$

Where $\tau_{\text {peak }}$ and $\mathrm{f}_{\text {peak }}$ are values at the local maxima on an F v. M" plot. A frequency shift of M" maxima will yield Arrhenius-like data plots using $\tau$ value. The decreasing size of the time constants in a Nyquist plot such as in figure 7 emulate the changes when subjected to increases in temperature (see supplemental information).

However literature to this point does not discern between $\tau_{\text {ion }}$ and $\tau_{\sigma}$. The difference can be explained by changes in mean proximity of the grains or the reduced inter-grain hopping distance, which in turn would increase both ion hopping frequency and ratio of complete hops from one site to another resulting from this reduction in activation energy while other conditions remain constant.

\subsection{X-ray diffraction studies}

Initially samples were examined for crystal structure which matched well to the product diffractograms provided from the manufacturer (Figure 3a). Subsequent diffractograms were taken for treated heat and/or pressure treated samples for comparison, to ensure crystal changes were not occurring for samples of interest. With the exception of twice annealed LATP and LLT, no crystal changes were observed. These are discussed further in the supplemental information.

\subsection{Annealing}

Annealing temperatures were determined by literature, and by experimentation. The aim was to relieve surface stress and optimize the ceramics. Annealing was conducted in an argon tube furnace, and in air for comparison. Some samples were reversibly reduced in argon indicated by a change in 
color, which was reversible by subsequent annealing in air. LATP when twice annealed in this fashion showed great improvement in conductivity. However, small crystal changes were observed, and were not investigated in this work. This is referred to hereafter as twice annealed LATP. Subsequent annealing in air resulted in LATP returning to a normal white color.

\section{Results}

\subsection{IS pressure studies}

In General, conductance increased for LAGP, LATP, and LLZO samples with increased pressure. We think that the intra-grain structure of the pressed sample collapses at some threshold pressure from mechanical failure, changing the system and thus the conductivity values. Unlike the other ceramics, LLTO samples decreased in conductance probably by affects described elsewhere ${ }^{40}$ and LLT did not have a reproducible response.

Using IS, grain boundary impedance $\left(\mathrm{Z}_{\mathrm{GB}}\right)$ is typically much larger by several orders of magnitude than the bulk impedance of the ceramic crystal $\left(Z_{B}\right)$. In these results the signal for bulk impedance (grains) are overwhelmed by the grain boundary impedance signal. In the high frequency range, the boundary is short circuited and capacitively conducting and negligible in magnitude, while the lower frequency $2^{\text {nd }}$ semicircle is in the time domain, and more closely resembles direct current in natures, mostly grain-to-grain conduction that varies with grain proximity ${ }^{42}$.

As the pressure increases within the confined space grains are forced closer, and rearrangement for stability until some upper mechanical limit is reached resulting in fracture and collapse of grain arrangement. Samples respond differently, many reaching a threshold where conductance abruptly increases, while others decrease.

This mechanical breakage resulting in smaller grains, and changing surface area (SA) the distance between grains (1) is dynamic in this pressurized system. For disordered systems which consist mostly of capacitive grain boundaries characterized by Sakamaki et al ${ }^{40}$, it is reasonable to modify the equation for capacitance to reflect changing conditions in this system (equation 6).

$$
d C=e_{0} \frac{\partial A}{\partial l} \text { or } d C=e_{0}\left(\frac{\partial A}{\partial l}\right)_{P}
$$

$\mathrm{C}=$ capacitance, $\mathrm{A}$ surface area, 1 (grain boundary) length and $\mathrm{P}$ pressure. 
The brick layer model is conceptually sound even in simple form, but has been updated ${ }^{41-45}$ in literature to account for grain size, geometry, percent of grain contact and so on, leaving the door open to perhaps model such systems better in the future.

With a grain boundary conductance that is much lower than the bulk as it is here, admittance equations may characterize a system in part (equation 11) when $\sigma_{b}>>\sigma_{g b}$ or 12 where $\sigma_{g b}>>\sigma_{g}$. $\Psi_{t}$ is total admittance, $\mathrm{x}$ is volume fraction, $\mathrm{g}$ is grain, gb grain boundary, for systems with known volumes of grain and grain boundaries. Pore space was measured using the Archimedes method, and it could possibly be used, in part, to determine the aforementioned volumes.

Sample pellets formed at maximum pressure (0.75 GPa typically) had a measured porosity between 18-30\%, without the use of binders. While not elastic, some relaxation of pelletized ceramics was expected and observed when released from the sample holder, therefore voids and pore space are larger in a formed pellet than when subjected to pressure.

While it is easy to make the assumption that the grains get closer together and more compact, and that conduction may improve, the underlying mechanisms are quite complex and interesting. Application of pressure up to $1.0 \mathrm{GPa}$ is not alone sufficient to cause a crystallographic change in the samples, so we can say that the changes in electrical measurements are a result of mechanical forces.

Twice annealed LATP conductance increased abruptly between 0.45 and $0.60 \mathrm{GPa}$ (figure $7 \mathrm{~F})$. it is speculated that the inter-grain structure in the sample cylinder collapses above $0.45 \mathrm{GPa}$ causing a settling of the LATP powder thus increasing surface contacts and reducing grain boundary regions. Example spectra LAGP as received M" vs. $\log \mathrm{F}$ indicates a lateral peak shift, indicating a frequency dependency and change in relaxation time. Magnitude of peaks indicates change in capacitance (eq. 2) LLTO versus LLTO annealed shows a decrease in $\sigma$ is observed for annealed samples. LLZO and LAGP likewise showed improved conductance under pressure, and with annealing. Additional $\varepsilon^{\prime}$ and $\mathrm{M}^{\prime \prime}$ vs. F trends are discussed in the supplementary information section.

\subsection{Scanning electron microscopy (SEM)}

Micrographs were taken at each experimental step when possible. Upon annealing, grains appear better defined. Calculations of average diameter indicate smaller average sizes for pressed, annealed, and annealed then pressed powders which were expected, likely due to mechanical stress and exploitation of surface cracks and imperfections by the heating process and/or pressure. 
Consequently this changes the overall grain size distribution, therefore a resulting change in grain and grain boundary surface areas. Most notably for LATP, an abrupt change in grain boundary impedance was noted probably due to collapse in intra-grain structure which occurred at about $0.45 \mathrm{GPa}$.

SEM images of samples illustrate what would be expected, that the grains are closer together with consolidation of grain boundaries which range are quite large, with voids for unconsolidated samples to closely packed grains for samples subjected to pressure to form pellets. Moreover some physical changes can be observed for the grains themselves for LAGP, LLZO and LATP. Visually the consistency of the ceramic powders differs from each treatment.

\subsection{TGA Analysis}

Powders as received were pressed into pellets to $0.75 \mathrm{GPa}$ prior to TGA analysis. All powders showed loss of weight due to increase of temperature, possibly due to retained water ${ }^{60-62}$, trapped gases ${ }^{25}$, decomposition ${ }^{63}$, or removal of synthesis residuals ${ }^{62}$. This alone could change the outcome for powders that were annealed then pressed compared to powders that were pressed, and then annealed because these contaminants could be reintroduced when pelletizing annealed powder.

Samples were ramped $10^{\circ} \mathrm{C} /$ min to annealing temperatures, held isothermally 60 minutes under nitrogen then cooled to $50^{\circ} \mathrm{C}$ (figure 9). At this step the flowing gas was switched (nitrogen, argon, or air) and held isothermally for 48 hours to observe weight changes summarized in Table 2. Improvement in conductance by gas replacement in the pores has been speculated elsewhere, and should not be ruled out. Breathing quality air ${ }^{A}$ was used in place of oxygen. Samples were allowed to stand 48 hours after cooling to observe weight change. The decrease in weight suggests possible consolidation of grains and decreased pore space of pellets, as well as an indication of pore space availability in each pressed ceramic pellet.

\section{Discussion}

In all ceramic systems described, grain boundary impedance dominates each system. This results in a rather low ionic conductivity overall for a disordered powder system. This is mitigated somewhat by the process of compressing the powders into a pellet, and by annealing the powders. Other possible methods would be ball milling which would be a consideration when using commercially available powders if a more uniform system is not available for purchase.

Without sintering, changes were observed at the grain boundary regions utilizing a novel application of IS such as modulus which has been in use but not a great deal over the years Modulus 
allows the observation of the grain boundary capacitance shift due to relaxation processes at the grain boundary ${ }^{64}$ in ways that are not possible with other methods. Nyquist plots while valuable, tend to dominate battery research where impedance measurements are concerned. Here, we associate electrochemical measurements with physical changes in the system which strongly suggests a connection between grain boundary conditions, pressure, and impedance based values.

Changes in electric modulus indicate capacitance changes pertaining to available surface areas, and proximity which could correlate to hopping frequency and relaxation times of ions during the hopping process. Likewise, this focuses more on the grain boundary as the bulk ceramics have been well studied in literature, neglecting somewhat the underlying mechanisms present in disordered solids. Changes in permittivity under pressure are indicative of polarization suggests a qualitative change in how easily a sample becomes polarized, which essentially a charge transfer through a material. This supports the observed changes in ion conductivity, as positive ions are transferred through as sample; this is countered by movement of negative charge. Our results do not indicate specific frequency dependence for permittivity, rather a general trend.

Here we have characterized and compared the changes in conductivity and other electrical properties of five commercially available lithium conducting ceramics, the interactions at the grain boundaries, and attempting to resolve these changes under different pressurized conditions without effecting a structural change in the bulk. A better understanding of the mechanisms involved is a step forward in realizing the production of solid state batteries, their practical and reliable use.

\section{Acknowledgments}

The authors would like to acknowledge members of The University of Tulsa Nanotechnology Institute for support and useful discussion from its members. JWO would like to thank the University of Tulsa Graduate School and Office of Research for travel funding for promoting this work. The work was supported in part by the Oklahoma Center for the Advancement of Science and Technology (OCAST) and NASA Grant No. NNX13AN01A issued through the NASA EPSCoR Program. 


\section{References}

1. Fergus, J. W., Ceramic and polymeric solid electrolytes for lithium-ion batteries. Journal of Power Sources 2010, 195 (15), 4554-4569.

2. Kumar, J.; Kichambare, P.; Rai, A. K.; Bhattacharya, R.; Rodrigues, S.; Subramanyam, G., A high performance ceramic-polymer separator for lithium batteries. Journal of Power Sources 2016, 301, 194-198.

3. Thangadurai, V.; Weppner, W., Recent progress in solid oxide and lithium ion conducting electrolytes research. Ionics 2006, 12 (1), 81-92.

4. Liu, W. Multilayer composite solid electrolytes for Lithium Ion Batteries. Ph.D., Syracuse University, 2016.

5. Knauth, P., Inorganic solid Li ion conductors: An overview. Solid State Ionics 2009, 180 (14-16), 911-916.

6. Rangasamy, E.; Wolfenstine, J.; Allen, J.; Sakamoto, J., The effect of 24c-site (A) cation substitution on the tetragonal-cubic phase transition in Li7-xLa3-xAxZr2O12 garnet-based ceramic electrolyte. Journal of Power Sources 2013, 230, 261-266.

7. Murugan, R.; Thangadurai, V.; Weppner, W., Fast Lithium lon Conduction in GarnetType Li7La3Zr2O12. Angewandte Chemie International Edition 2007, 46 (41), 7778-7781.

8. Mariappan, C. R.; Gnanasekar, K. I.; Jayaraman, V.; Gnanasekaran, T., Lithium ion conduction in Li5La3Ta2O12 and Li7La3Ta2O13 garnet-type materials. Journal of Electroceramics 2013, 30 (4), 258-265.

9. Murugan, R.; Thangadurai, V.; Weppner, W., Effect of lithium ion content on the lithium ion conductivity of the garnet-like structure Li5+xBaLa2Ta2O11.5+0.5x $(x=0-2)$. Applied Physics A 2008, 91 (4), 615-620.

10. Kosova, N. V.; Devyatkina, E. T.; Stepanov, A. P.; Buzlukov, A. L., Lithium conductivity and lithium diffusion in NASICON-type Li1+xTi2-xAIx(PO4)3 $(x=0 ; 0.3)$ prepared by mechanical activation. Ionics 2008, 14 (4), 303-311.

11. Thangadurai, V.; Weppner, W., Effect of B-site substitution of (Li,La)TiO3 perovskites by di-, tri-, tetra- and hexavalent metal ions on the lithium ion conductivity (E4). lonics 2000, 6 (1), 70-77.

12. Evgenij Barsoukov, J. R. M., Impedance Spectroscopy: Theory, Experiment, and applications 2nd edition. 2005 ed.; John Wiliey \& Sons: 2005; p 581.

13. Murugan, R.; Thangadurai, V.; Weppner, W., Lithium ion conductivity of Li5+x Ba x La3-x Ta2O12 $(x=0-2)$ with garnet-related structure in dependence of the barium content. lonics 2007, 13 (4), 195-203.

14. Kothari, D. H.; Kanchan, D. K., Study of Li+ conduction in Li1.3AI0.3-xScxTi1.7(PO4)3 ( $x=0.01,0.03,0.05$ and 0.07) NASICON ceramic compound. Physica B: Condensed Matter 2016, 494, 20-25.

15. Irvine, J. T. S.; Sinclair, D. C.; West, A. R., Electroceramics: Characterization by Impedance Spectroscopy. Advanced Materials 1990, 2 (3), 132-138.

16. Hagman, L.-O. K., Peder, The Crystal Structure of NaMes ${ }^{\mathrm{IV}}\left(\mathrm{PO}_{4}\right)_{3}: \mathrm{Me}^{\mathrm{IV}}=\mathrm{Ge}, \mathrm{Ti}, \mathrm{Zr}$. Acta Chemica Scandinavica 1968, 22, 1822-1832.

17. Aono, H.; Sugimoto, E.; Sadaoka, Y.; Imanaka, N.; Adachi, G. y., lonic Conductivity of the Lithium Titanium Phosphate (Li1 + X M X Ti2 - X

( PO 4 ) $3, \mathrm{M}=\mathrm{Al}, \mathrm{Sc}, \mathrm{Y}$, and La) Systems. Journal of The Electrochemical Society 1989, $136(2), 590-591$.

18. Li, S.-C.; Lin, Z.-X., Phase relationship and ionic conductivity of Li1+xTi2-xInxP3O12 system. Solid State lonics 1983, 9-10, Part 2, 835-837. 
19. Monchak, M.; Hupfer, T.; Senyshyn, A.; Boysen, H.; Chernyshov, D.; Hansen, T.; Schell, K. G.; Bucharsky, E. C.; Hoffmann, M. J.; Ehrenberg, H., Lithium Diffusion Pathway in Li1.3AI0.3Ti1.7(PO4)3 (LATP) Superionic Conductor. Inorganic Chemistry 2016, 55 (6), 2941-2945.

20. Zhao, E.; Ma, F.; Guo, Y.; Jin, Y., Stable LATP/LAGP double-layer solid electrolyte prepared via a simple dry-pressing method for solid state lithium ion batteries. RSC Advances 2016, 6 (95), 92579-92585.

21. Aono, H.; Sugimoto, E.; Sadaoka, Y., Electrical properties of sintered lithium titanium phosphate ceramics (Li1+xMxTi2-x (PO4)3, M3+= Al3+, Sc3+, or Y3+). Chem Lett 1990, 10.

22. Zu-xiang, L.; Hui-jun, Y.; Shi-chun, L.; Shun-bao, T., Phase relationship and electrical conductivity of Li1+xTi2-xGaxP3O12 and Li1+2xTi2-xMgxP3O12 systems. Solid State lonics 1986, 18-19, Part 1, 549-552.

23. Duluard, S.; Paillassa, A.; Puech, L.; Vinatier, P.; Turq, V.; Rozier, P.; Lenormand, P.; Taberna, P.-L.; Simon, P.; Ansart, F., Lithium conducting solid electrolyte Li1.3Al0.3Ti1.7(PO4)3 obtained via solution chemistry. Journal of the European Ceramic Society 2013, 33 (6), 1145-1153.

24. $\quad$ Song, W.; Ji, X.; Wu, Z.; Zhu, Y.; Yang, Y.; Chen, J.; Jing, M.; Li, F.; Banks, C. E., First exploration of $\mathrm{Na}$-ion migration pathways in the NASICON structure Na3V2(PO4)3. Journal of Materials Chemistry A 2014, 2 (15), 5358-5362.

25. Kumar, B.; Kichambare, P.; Rodrigues, S.; Kumar, J.; Keil, R. G., Lisicon GlassCeramics Mediated Catalysis of Oxygen Reduction. Electrochemical and Solid-State Letters 2011, 14 (6), A97-A99.

26. Berbano, S. S.; Guo, J.; Guo, H.; Lanagan, M. T.; Randall, C. A., Cold sintering process of Li1.5Al0.5Ge1.5(PO4)3 solid electrolyte. Journal of the American Ceramic Society 2017, 100 (5), 2123-2135.

27. Kotobuki, M.; Suzuki, Y.; Munakata, H.; Kanamura, K.; Sato, Y.; Yamamoto, K.; Yoshida, T., Effect of sol composition on solid electrode/solid electrolyte interface for allsolid-state lithium ion battery. Electrochimica Acta 2011, 56 (3), 1023-1029.

28. Aono, H.; Sugimoto, E.; Sadaoka, Y.; Imanaka, N.; Adachi, G. y., lonic Conductivity of Solid Electrolytes Based on Lithium Titanium Phosphate. Journal of The Electrochemical Society 1990, $137(4), 1023-1027$.

29. Düvel, A.; Kuhn, A.; Robben, L.; Wilkening, M.; Heitjans, P., Mechanosynthesis of Solid Electrolytes: Preparation, Characterization, and Li Ion Transport Properties of GarnetType Al-Doped Li7La3Zr2O12 Crystallizing with Cubic Symmetry. The Journal of Physical Chemistry C 2012, 116 (29), 15192-15202.

30. Redhammer, G. J.; Rettenwander, D.; Pristat, S.; Dashjav, E.; Kumar, C. M. N.; Topa, D.; Tietz, F., A single crystal X-ray and powder neutron diffraction study on NASICON-type Li1+xAlxTi2-x(PO4)3 $(0 \leq x \leq 0.5)$ crystals: Implications on ionic conductivity. Solid State Sciences 2016, 60, 99-107.

31. Kothari, D. H.; Kanchan, D. K., Effect of doping of trivalent cations Ga3+, Sc3+, Y3+ in Li1.3Al0.3Ti1.7 (PO4)3 (LATP) system on Li+ ion conductivity. Physica B: Condensed Matter 2016, 501, 90-94.

32. Aguesse, F.; Manalastas, W.; Buannic, L.; Del Amo, J. M. L.; Singh, G.; Llordés, A.; Kilner, J., Investigating the dendritic growth during full cell cycling of garnet electrolyte in direct contact with Li metal. ACS Applied Materials and Interfaces 2017, 9 (4), 3808-3816. 
33. van Den Broek, J.; Rupp, J. L. M.; Afyon, S., Boosting the electrochemical

performance of Li-garnet based all-solid-state batteries with Li4Ti5O12 electrode: Routes to cheap and large scale ceramic processing. Journal of Electroceramics 2017, 1-7.

34. Tsai, C.-L.; Roddatis, V.; Chandran, C. V.; Ma, Q.; Uhlenbruck, S.; Bram, M.; Heitjans, P.; Guillon, O., Li7La3Zr2O12 Interface Modification for Li Dendrite Prevention. ACS Applied Materials \& Interfaces 2016, 8 (16), 10617-10626.

35. El Shinawi, H.; Janek, J., Stabilization of cubic lithium-stuffed garnets of the type "Li7La3Zr2O12" by addition of gallium. Journal of Power Sources 2013, 225, 13-19.

36. Allen, J. L.; Wolfenstine, J.; Rangasamy, E.; Sakamoto, J., Effect of substitution (Ta, $\mathrm{Al}, \mathrm{Ga})$ on the conductivity of Li7La3Zr2O12. Journal of Power Sources 2012, 206 (Supplement C), 315-319.

37. Inaguma, Y.; Liquan, C.; Itoh, M.; Nakamura, T.; Uchida, T.; Ikuta, H.; Wakihara, M., High ionic conductivity in lithium lanthanum titanate. Solid State Communications 1993, 86 (10), 689-693.

38. Ahmad, M. M., Lithium ionic conduction and relaxation dynamics of spark plasma sintered Li5La3Ta2O12 garnet nanoceramics. Nanoscale Research Letters 2015, 10 (1), 110.

39. Onishi, H.; Takai, S.; Yabutsuka, T.; Yao, T., Synthesis and Electrochemical properties of LATP-LLTO lithium ion conductive composites. Electrochemistry 2016, 84 (12), 967-970.

40. Sakamaki, R.; Sakamoto, N.; Fujiki, H.; Kaneko, N. H., Local DC electrical properties of La2/3-xLi3xTiO3 grain boundaries. Nippon Seramikkusu Kyokai Gakujutsu

Ronbunshi/Journal of the Ceramic Society of Japan 2016, 124 (9), 907-910.

41. Maier, J., On the Conductivity of Polycrystalline Materials. Berichte der Bunsengesellschaft für physikalische Chemie 1986, 90 (1), 26-33.

42. Fleig, J.; Maier, J., Finite-Element Calculations on the Impedance of Electroceramics with Highly Resistive Grain Boundaries: I, Laterally Inhomogeneous Grain Boundaries. Journal of the American Ceramic Society 1999, 82 (12), 3485-3493.

43. Bouchet, R.; Knauth, P.; Laugier, J.-M., Theoretical analysis of the impedance spectra of electroceramics Part 2: isotropic grain boundaries. Journal of Electroceramics 2006, 16 (3), 229-238.

44. Kidner, N. J.; Perry, N. H.; Mason, T. O.; Garboczi, E. J., The Brick Layer Model Revisited: Introducing the Nano-Grain Composite Model. Journal of the American Ceramic Society 2008, 91 (6), 1733-1746.

45. Erdoğan, S. T., Simple Estimation of the Surface Area of Irregular 3D Particles. Journal of Materials in Civil Engineering 2016, 28 (8), 04016062.

46. Inaguma, Y.; Nakashima, M., A rechargeable lithium-air battery using a lithium ionconducting lanthanum lithium titanate ceramics as an electrolyte separator. Journal of Power Sources 2013, 228, 250-255.

47. Kalnaus, S.; Tenhaeff, W. E.; Sakamoto, J.; Sabau, A. S.; Daniel, C.; Dudney, N. J., Analysis of composite electrolytes with sintered reinforcement structure for energy storage applications. Journal of Power Sources 2013, 241, 178-185.

48. Hiratani, M.; Miyauchi, K.; Kudo, T., Electrode reaction at the interface between a lithium anode and a solid electrolyte. Solid State lonics 1988, 28-30, 1431-1435.

49. Jackman, S. D.; Cutler, R. A., Effect of microcracking on ionic conductivity in LATP. Journal of Power Sources 2012, 218, 65-72. 
50. Schell, K. G.; Bucharsky, E. C.; Lemke, F.; Hoffmann, M. J., Effect of calcination conditions on lithium conductivity in Li1.3Ti1.7AI0.3(PO4)3 prepared by sol-gel route. Ionics 2016, 1-7.

51. Tian, F.; Ohki, Y., Electric modulus powerful tool for analyzing dielectric behavior. IEEE Transactions on Dielectrics and Electrical Insulation 2014, 21 (3), 929-931. 52. Vasoya, N. H.; Jha, P. K.; Saija, K. G.; Dolia, S. N.; Zankat, K. B.; Modi, K. B., Electric Modulus, Scaling and Modeling of Dielectric Properties for Mn2+-Si4+ Cosubstituted Mn-Zn Ferrites. Journal of Electronic Materials 2016, 45 (2), 917-927. 53. Fuqiang Tian; Ohki, Y., Electric Modulus a Powerful Tool for Analyzing Dielectric Behavior. IEEE Transactions on Dielectrics and Electrical Insulation 2014, 21 (3), 14331433.

54. Belal Hossen, M.; Akther Hossain, A. K. M., Complex impedance and electric modulus studies of magnetic ceramic Ni0.27Cu0.10Zn0.63Fe2O4. Journal of Advanced Ceramics 2015, 4 (3), 217-225.

55. Behera, A. K.; Mohanty, N. K.; Satpathy, S. K.; Behera, B.; Nayak, P., Investigation of complex impedance and modulus properties of $\mathrm{Nd}$ doped $0.5 \mathrm{BiFeO}-0.5 \mathrm{PbTiO} 3$ multiferroic Composites. Central European Journal of Physics 2014, 12 (12), 851-861. 56. Liu, J.; Duan, C.-G.; Yin, W.-G.; Mei, W. N.; Smith, R. W.; Hardy, J. R., Dielectric permittivity and electric modulus in Bi2Ti4O11. The Journal of Chemical Physics 2003, 119 (5), 2812-2819.

57. Ngai, K. L.; León, C., Relating macroscopic electrical relaxation to microscopic movements of the ions in ionically conducting materials by theory and experiment. Physical Review B 1999, 60 (13), 9396-9405.

58. Wang, Y. H., Kun; Zu, Chengkui; Chen, Jiang, Study on crystallization kinetics of Li2)-Al2O3-TiO2-P2O5 glass by non-isothermal techniques. Advanced Materials Research 2012, 535-537, 1629-1633.

59. Jeff Wolfenstinea, E. R., Jan L. Allen, Jeffrey Sakamoto, High conductivity of dense tetragonal Li7La3Zr2O12. Journal of Power Sources (2012), 208, 193-196.

60. Mazumdar, D.; Bose, D. N.; Mukherjee, L. L.; Basu, A.; Bose, M., 7Li NMR and related studies in LISICON system. Materials Research Bulletin 1983, 18 (1), 79-86.

61. Waetzig, K.; Rost, A.; Langklotz, U.; Matthey, B.; Schilm, J., An explanation of the microcrack formation in Li1.3Al0.3Ti1.7(PO4)3 ceramics. Journal of the European Ceramic Society 2016, 36 (8), 1995-2001.

62. Belam, W., Sol-gel chemistry synthesis and DTA-TGA, XRPD, SIC and 7Li, 31P, 29Si MAS-NMR studies on the Li-NASICON Li3Zr2-ySi2-4yP1+4yO12 $(0 \leqslant y \leqslant 0.5)$ system. Journal of Alloys and Compounds 2013, 551, 267-273.

63. Zangina, T.; Hassan, J.; Matori, K. A.; Azis, R. a. S.; Ahmadu, U.; See, A., Sintering behavior, ac conductivity and dielectric relaxation of Li1.3Ti1.7Al0.3(PO4)3 NASICON compound. Results in Physics 2016, 6, 719-725.

64. Feliciangeli, M. C.; Rossi, M. C.; Conte, G., An admittance spectroscopy study of grain and grain boundary of diamond. Diamond and Related Materials 2007, 16 (4), $930-$ 934. 
Figures

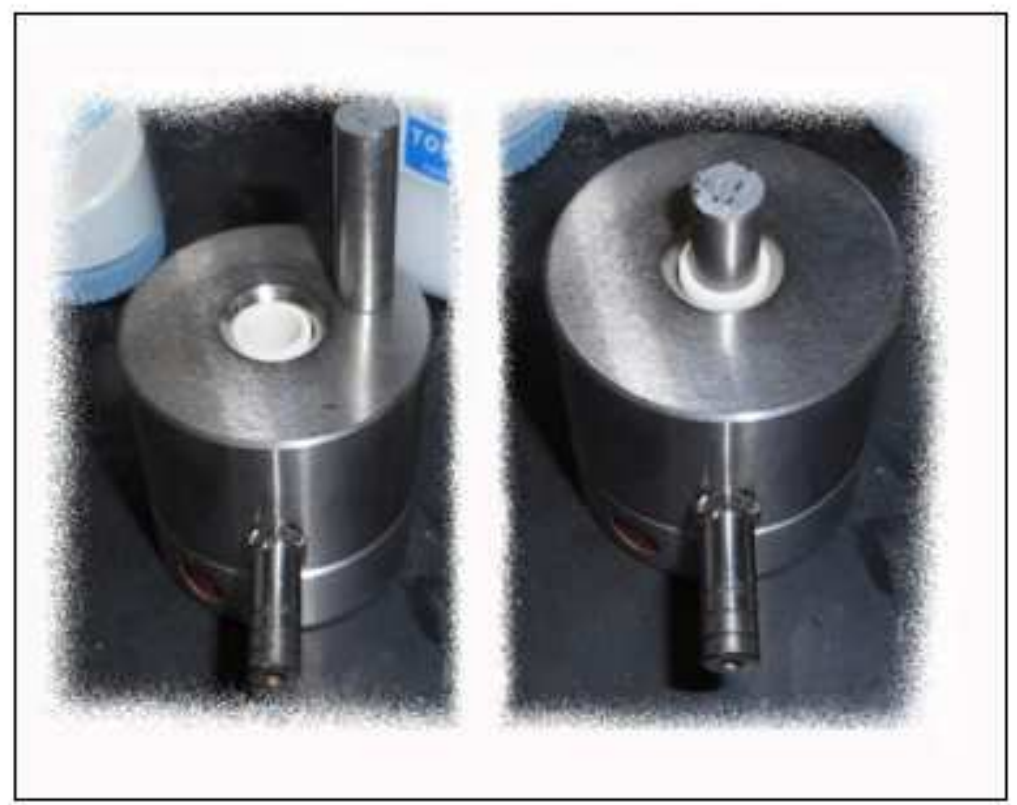

Figure 1

The steel shaft is inserted into the tube, and O-rings were used on both shaft and tube to facilitate vacuum 


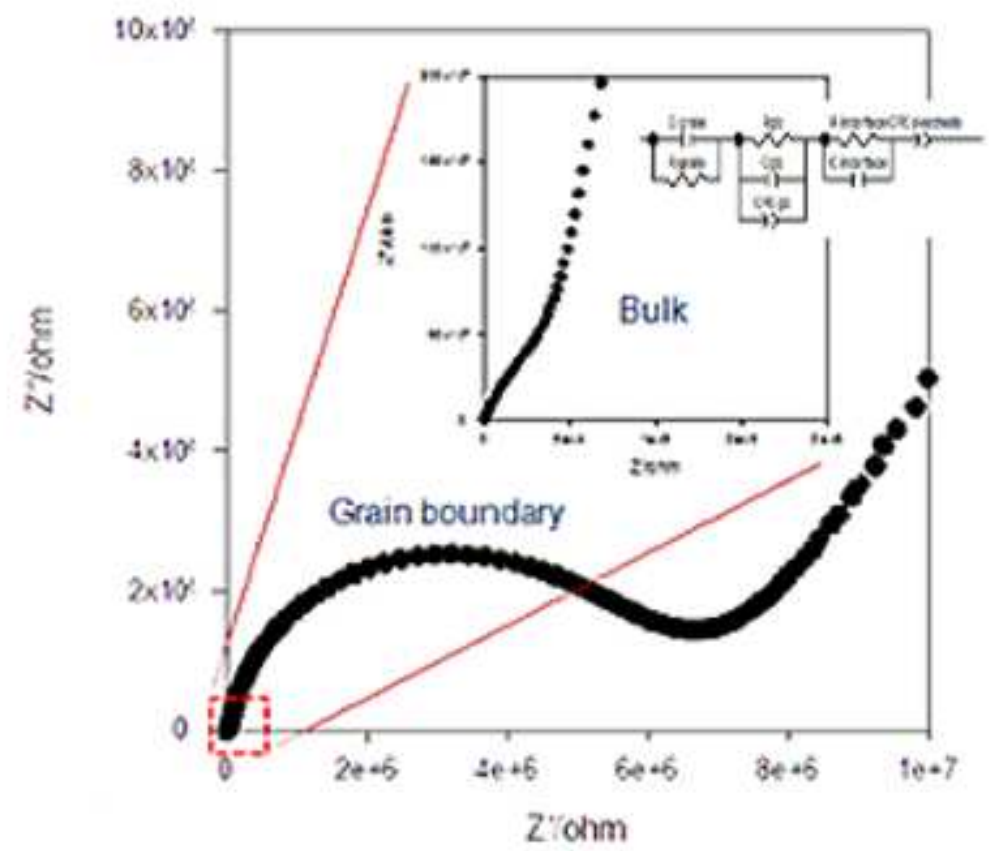

Figure 2

\section{Figure 2}

In part due to scaling, Nyquist plots can be inconvenient to discern phases as the semi circles

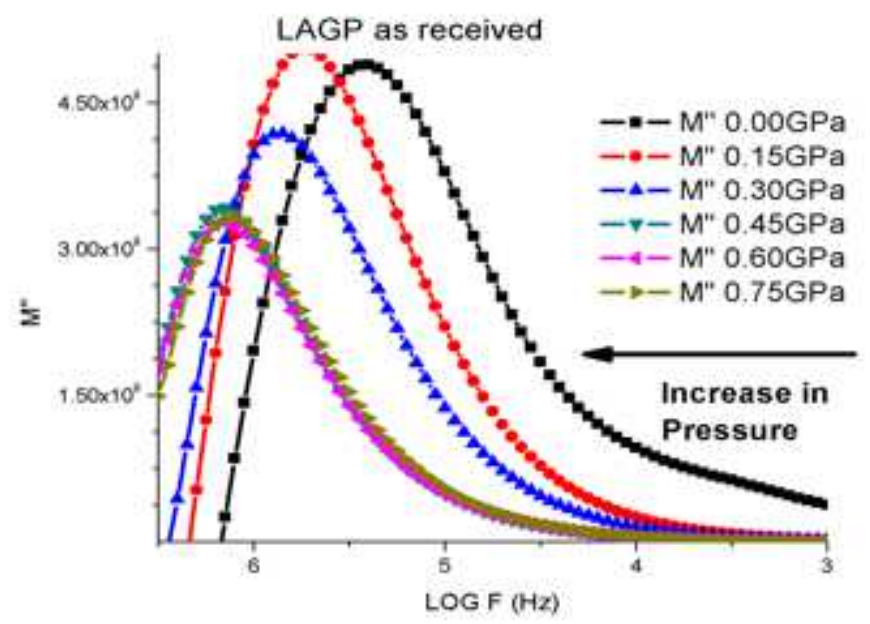

Figure 3 
Figure 3

LAGP (as received) pressed from 0.00-0.75GPa)

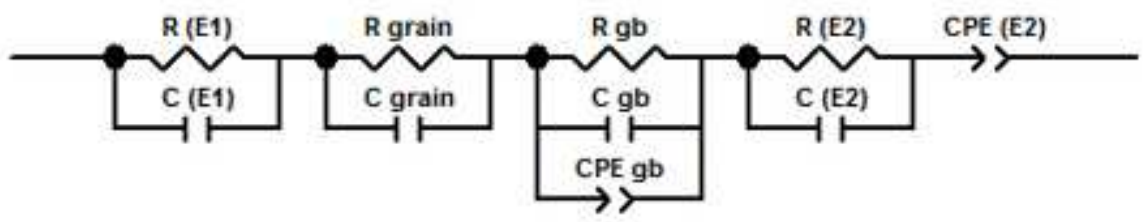

Figure 4

equivalent circuit model

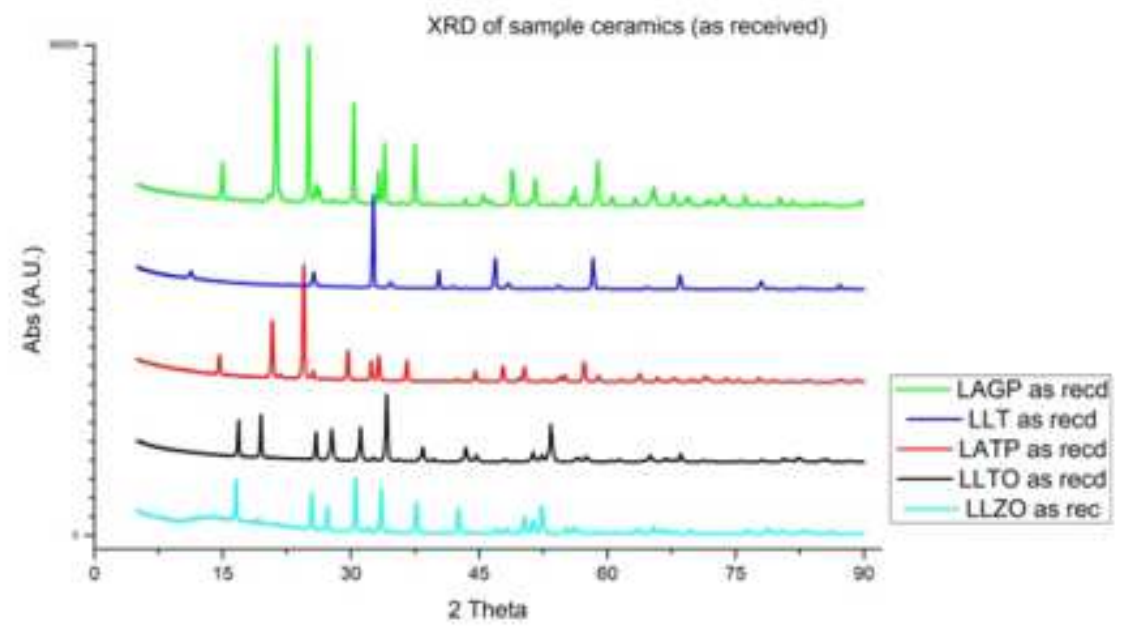

Figure 5

comparison of ceramic powers used

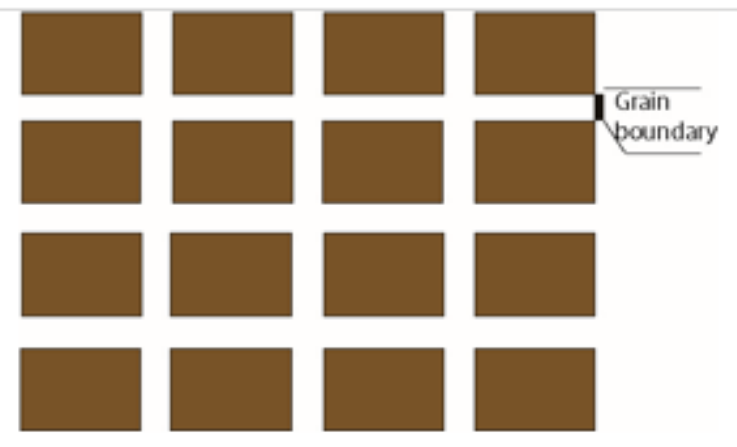

Unconsolidated grains without pressure

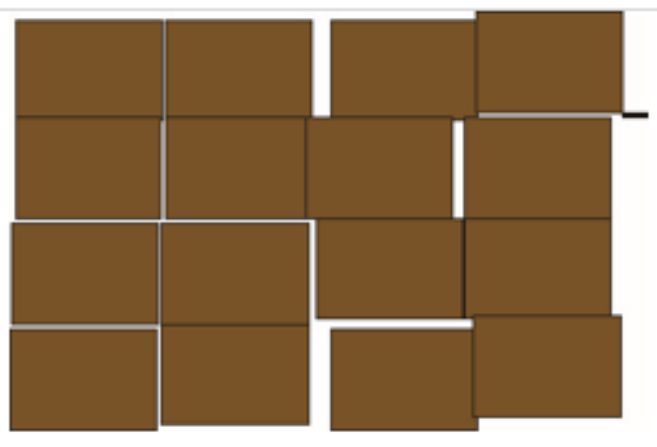

Random rearrangement while under pressure 
Figure 6

simplified brick and mortar model

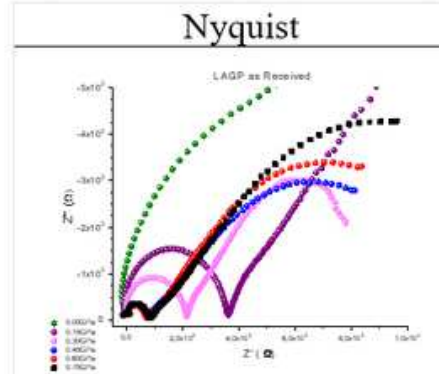

A. LAGP as received

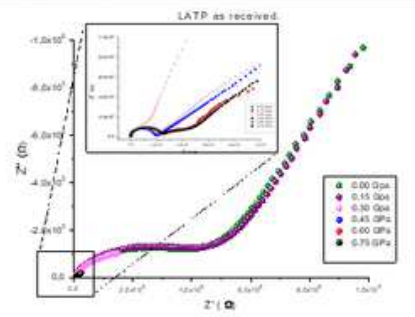

E. LATP as received

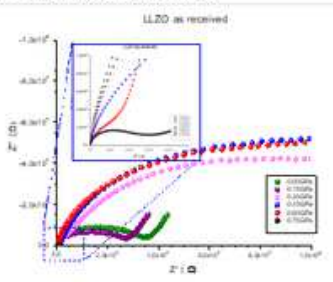

I. LLZO as received

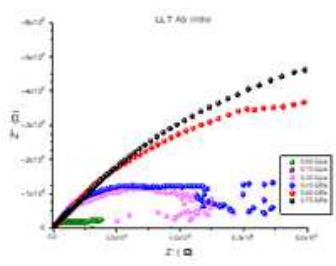

M. LLT as received

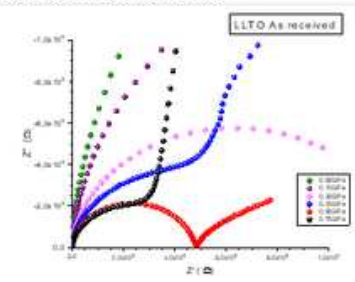

Q. LLTO as received
Conductance

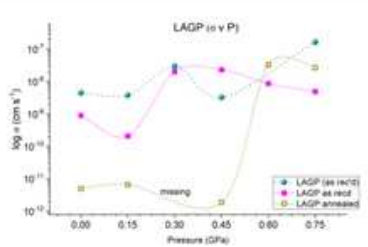

B. LAGP vs LAGP annealed

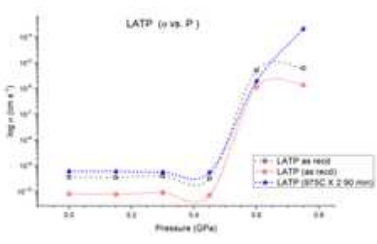

F. LATP vs LATP annealed

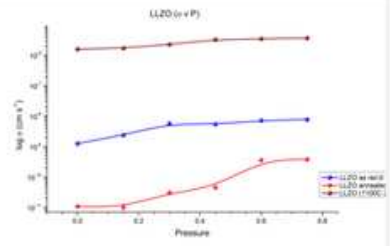

J. LLZO vs LLZO annealed

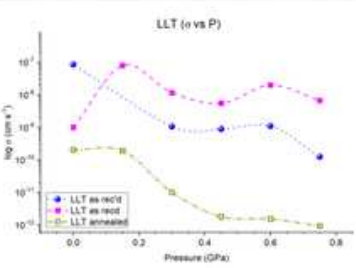

N. LLT versus LLT annealed

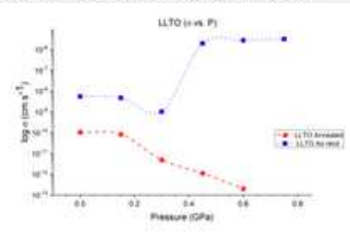

R. LLTO versus LLTO annealed
$\mathrm{M}^{\prime \prime}$ vs $\log \mathrm{F}$

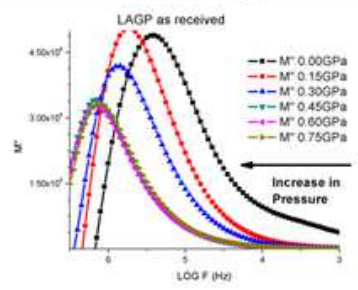

C. LAGP as received

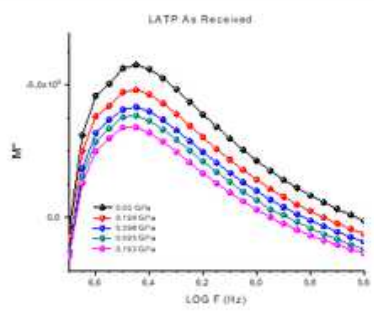

G. LATP

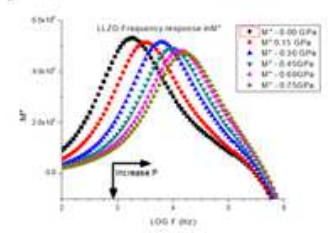

K. LLZO

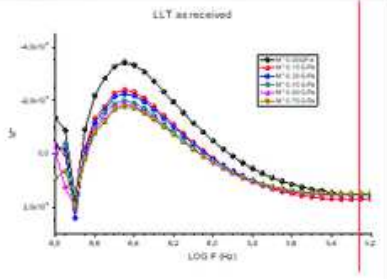

O. LLT

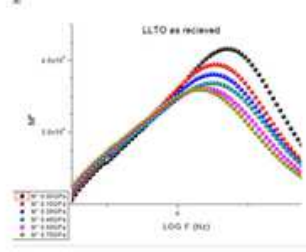

S. LLTO $\varepsilon$ vs F

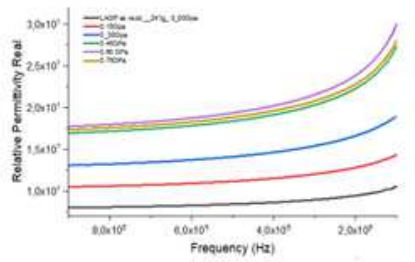

D. LAGP

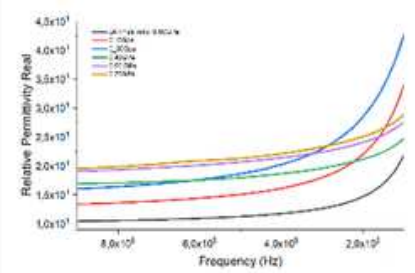

H. LATP

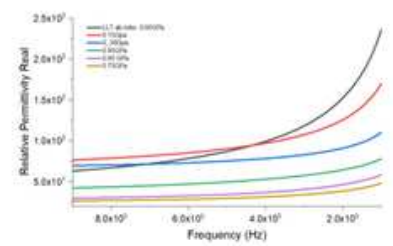

L. LLZO

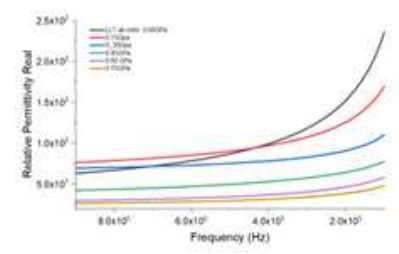

P. LLT

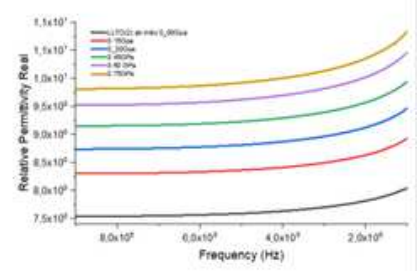

T. LLTO

\section{Figure 7}

from left to right for samples as received (exept conductance plot): Nyquist plot, sample counductance under pressure, electric modulus changes under pressure, and permittivity changes under pressure for 


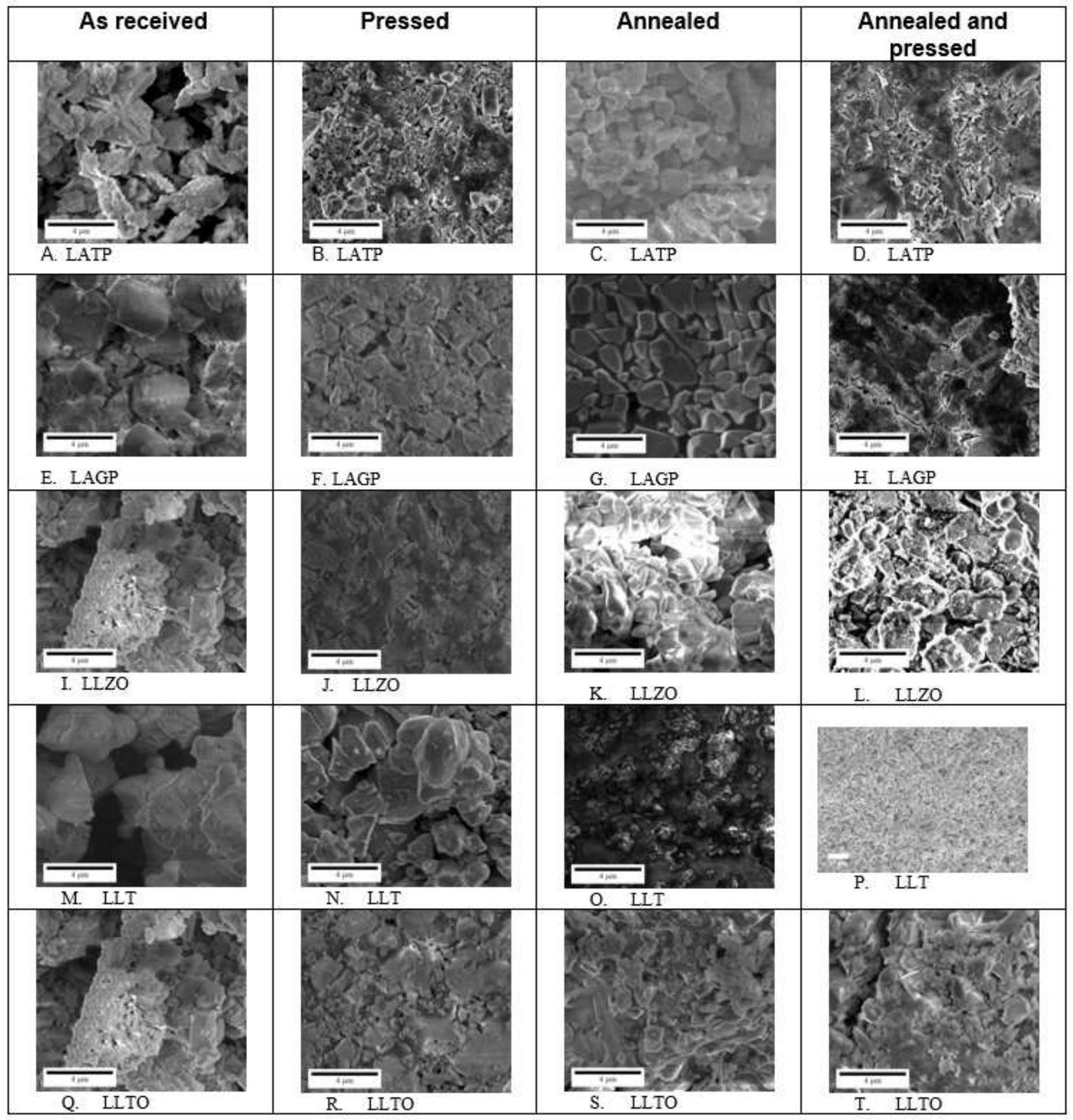

Figure 8

SEM for each ceramic tested from left to right: as received, pressed, annealed (not pressed), and annealed and pressed. 


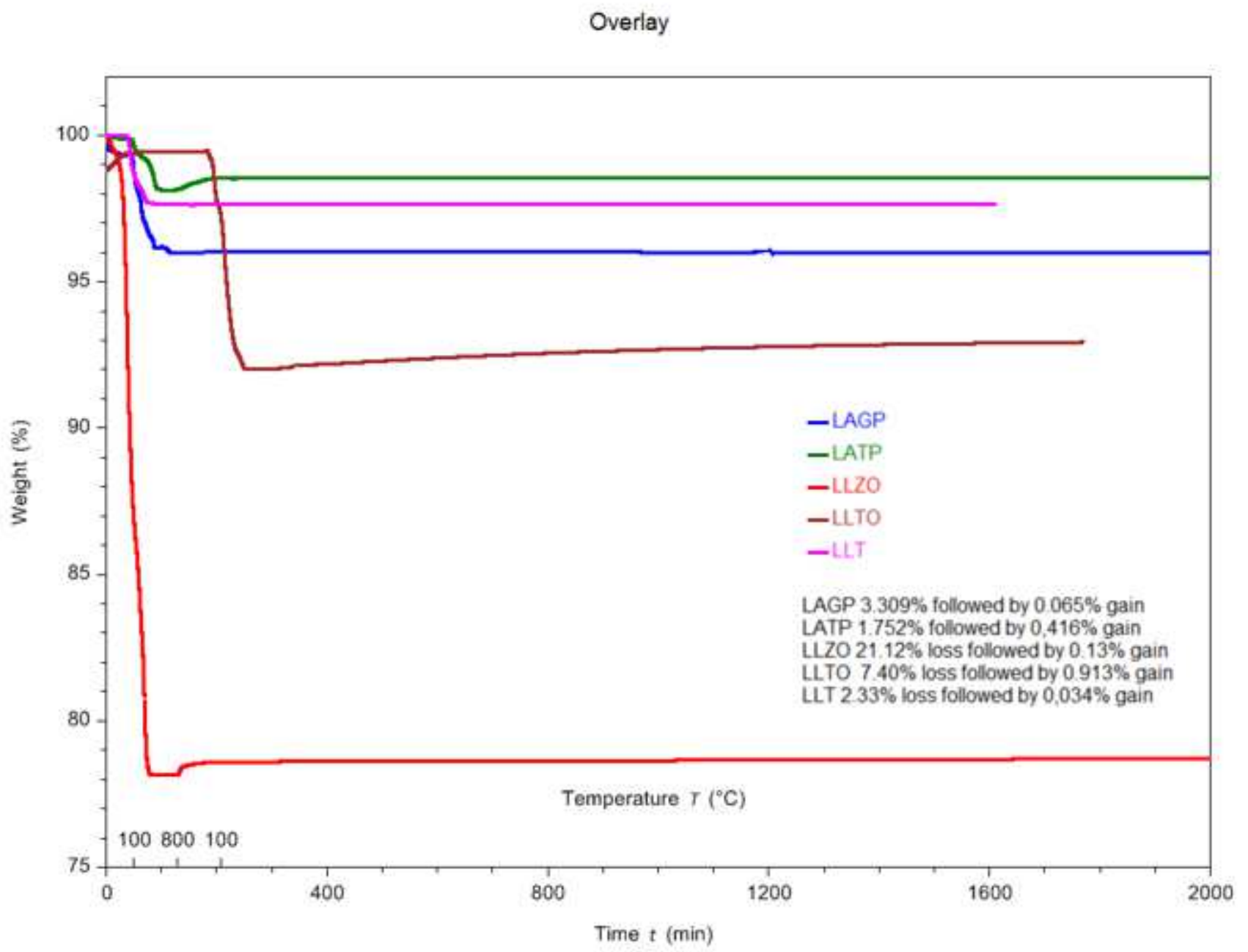

Figure 9

select TGA values for ceramic pellets

\section{Supplementary Files}

This is a list of supplementary files associated with this preprint. Click to download.

- Tables.pdf

- CeramicsSupplementaryinfoJWOFInal.docx 